\title{
ANTISENSE OLIGONUCLEOTIDES AND RADIONUCLIDES - PERSPECTIVES
}

\author{
Katya B. Popova, Maria Mincheva, Maria Yavahchova, Nikolay Goutev, Dimitar Tonev
}

\begin{abstract}
Over the past decade, antisense technology has successfully established itself as an entirely innovative platform for research and creation of new therapies. Significant advances in the design of antisense oligonucleotides, as well as a deeper understanding of their mechanisms of action, have led to their successful clinical application in many RNA-targeted therapies. In addition, their potential for in vivo imaging by radiolabeling has been identified. Here are discussed the prospects for the use of antisense oligonucleotides in nuclear medicine and highlighted some of the advantages and disadvantages of labelling them with radionuclides.
\end{abstract}

Keywords: Antisense oligonucleotides, radionuclides, nuclear medicine, radiolabeling, advantages, disadvantages.

\section{INTRODUCTION}

The last 20 years have seen increased progress in the field of antisense technology. This technology provides an opportunity to manipulate gene expression at the RNA level through the socalled antisense oligonucleotides. Antisense oligonucleotides are DNA or RNA sequences whose length usually varies between 20 and $25 \mathrm{nt}$ [1]. They bind to specific RNA targets according to the canonical Watson-Crick base pairing and cause modulation of gene expression. This is achieved through two main mechanisms of action - RNase $\mathrm{H}$-dependent and RNase $\mathrm{H}$-independent mechanism, which are determined by the type of applied chemical modifications of antisense oligonucleotides [2,3]. Due to the wide variety of applied chemical modifications and the ability of the antisense oligonucleotides to target virtually every transcribed RNA molecule in the human body, their application has begun to be studied intensively in various biological fields. This has led to the successful development and approval of 7 drugs based on ASOs [4-6].At the same time, in the scientific literature there is experimental evidence of their successful use not only as antiviral [7-9] and antiparasitic [10] agents but also as therapeutics in the field of inflammatory diseases [11], diseases of the central nervous system [12, 13], bacterial infections [14, 15], and cancer [16-18]. That is why the question arises what the prospects for antisense oligonucleotides in the field of nuclear medicine are [19]?

Antisense oligonucleotides can be used in combination with radiopharmaceuticals (radionuclides) for the so-called antisense imaging [20-23]. This allows in vivo detection and monitoring of molecular events such as tracking the uptake of ASO and its passage through various barriers in the human body, as well as the study of gene expression of its targets in the relevant tissues and organs. The advantage of antisense imaging lies not only in the specificity of the targets in ASO technology but also in the non-invasive nature of imaging methods for radionuclide detection. For this purpose, both classical nuclear medical imaging methods such as positron emission tomography (PET) and single-photon emission computed tomography (SPECT), as well as hybrid imaging methods - PET-CT and SPECT-CT are used. They give high sensitivity and selectivity of images, and their combination with computer tomography (CT) gives a complex image of the target organ, combining functional information with detailed morphological information from computed tomography [24].Despite the constant improvements in the imaging technology, the ASO modifications applied, and the large selection of radionuclides for conjugation, there are still some challenges in the use of radiolabeled ASOs. In this article, we briefly discuss some of the strengths and weaknesses of labelling ASOs with radionuclides.

\section{CHEMICAL MODIFICATIONS}

Designing ASOs with high specificity and effectiveness is not easy. It requires thorough bioinformatics analysis for suitable RNA targets and the biochemical pathways they are part of [25, 26]. Furthermore, the secondary structure of the potential RNA target has to be determined, which is achievable by the use of various available bioinformatic databases and software tools such as Ensembl, PFRED, Mfold, RNAfold web server and etc [27-29]. Once a suitable region within the RNA molecule is chosen, it is not enough to synthesize an ASO with a reverse complementary sequence. In their native form, antisense oligonucleotides would not be suitable for use in clinical practice, as they are an easy target for enzymatic degradation by nucleases. Moreover, they have impaired target specificity and affinity for binding to non-specific 
proteins, resulting in undesired cytotoxicity. In addition, their uptake and distribution in the human body, as well as their successful attainment and entry into target cells, is hampered by several tissue barriers explained later in this paper. Therefore, in practice, it is necessary to use chemical modifications that significantly improve the pharmacokinetic and pharmacodynamic characteristics of ASOs. According to the type of ASO modifications applied, three generations of ASOs can be distinguished (Fig. 1)[2].
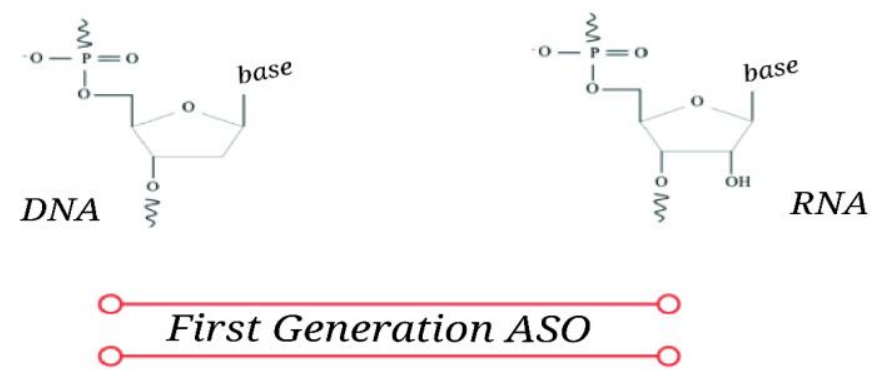

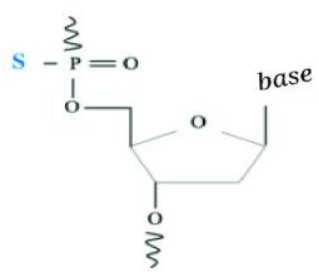

Phosphorothioate (PS)

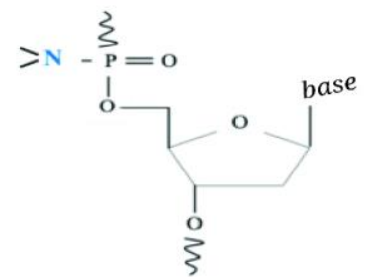

Phosphoroamidate
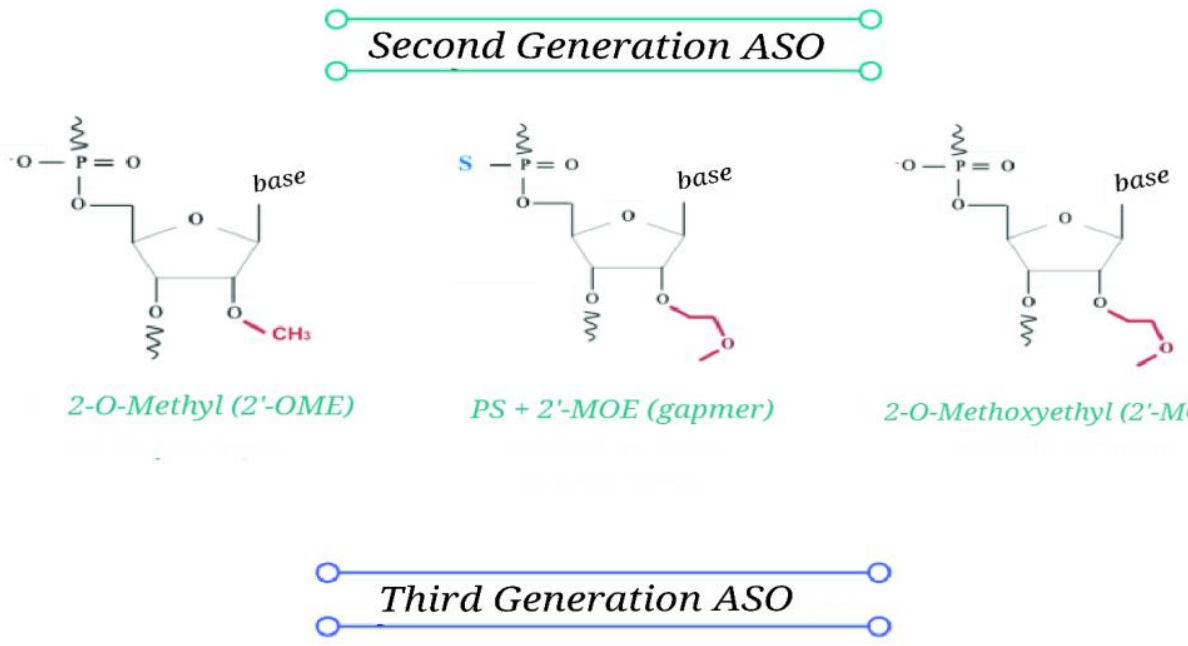

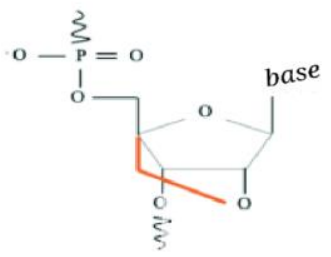

Locked nucleic acid (LNA)

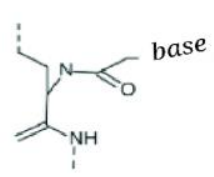

Peptide nucleic acid (PNA)

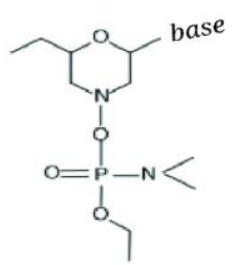

Morpholino phosphoroamidate oligomer (MFO)

Fig. 1. Chemical modifications of antisense oligonucleotides. 
The first generation of ASOs is characterized by modifications applied to one of the non-bridged oxygen atoms in the phosphodiester bond [2].This type of modification replaces it with another chemical element or chemical group such as sulfur (phosphorothioate modification) or amines (phosphoramidate modification). These modifications successfully increase the resistance of ASOs to nucleases and prolong their life in vivo, but their affinity for complementary binding to the targeted mRNA remains weak compared to subsequent generations. The main characteristic of the first generation is that when the ASO binds to the target mRNA, the enzyme RNase $\mathrm{H}$ is activated, which recognizes the double-stranded region (duplex) formed and degrades the target mRNA without affecting the ASO [2]. However, this mechanism of action leads to a significant decrease in the target mRNA in the cells, which makes it unsuitable for antisense imaging. Instead, the use of next-generation modifications is recommended to preserve mRNA and consequently, achieve longer retention of the radiopharmaceuticals in the target cells.

The second generation of ASOs is characterized by 2'-alkyl modifications such as 2'-O-methyl and 2'$O$-methoxyethyl modifications [30]. They successfully correct the shortcomings of the previous generation and increase the affinity for ASO binding to the RNA target. In addition, the stability of the resulting DNA-RNA or RNA-RNA duplex is greater [30], the presence of ASO in the tissues is increased and lower concentration is required to achieve a therapeutic effect [30]. Pointed out disadvantage of these modifications is that they do not activate RNase $\mathrm{H}$, which necessitates the use of the so-called gapmer structure [31]. Firstgeneration modifications are applied in the central part of the oligomer, and second-generation modifications are applied at both ends of the oligomer. Thus, ASO has increased specificity and nuclease resistance, in combination with activation of the RNase $\mathrm{H}$ mechanism of enzyme degradation. However, the RNAase H-dependent mechanism is required only when ASOs are required to inhibit a particular mRNA. Here, as already mentioned, this mechanism is not needed, as it would only further complicate the reading of the image.

The third generation is characterized by various changes in the chemical structure of the furanose ring and/or in the phosphate linkages [2]. Third generation ASOs have even stronger resistance to nuclease degradation $[2,30,31]$ and stronger stability of the formed duplex $[2,31]$. Some of the most popular examples of ASOs of this generation are the locked nucleic acid (LNA) [2], phosphorodiamidate morpholino oligomers (PMO) [2], and peptide nucleic acid (PNA) [2]. Among them, both PMO and PNA are hydrophobic and have a neutral charge [32, 33], unlike previous generations, which were characterized by a negative charge [2]. When radiolabelling is required for ASOs from this generation or the previous one, modifications are usually made to the $5^{\prime}$ or $3^{\prime}$ end of the antisense oligomer. A typical example of such a modification is the coupling of the oligomer to a primary amine [34].

\section{DISADVANTAGES AND OBSTACLES}

It is possible that ASOs do not exhibit the same properties in vivo compared to those shown in in vitro studies [35]. This is because ASOs must be able to cross many barriers in the human body to successfully reach the target tissues and cells. Typical examples of this are the blood-brain barrier [12, 36-38], the reticuloendothelial system [39], the vascular endothelial barrier [39], and renal excretion [39]. Therefore, not only the applied modifications of the ASOs should be taken into consideration, but also the form in which they will be taken in vivo. For example, with parenteral injection, ASOs are absorbed from the bloodstream within minutes to several hours [40, 41], but most of them cannot cross the blood-brain barrier [39]. This is an important hindrance for the application of radiolabeled ASOs in nuclear medicine, especially in the cases they are needed for imaging a patient's brain. In such cases it is usually recommended to use 3 local routes of transmission - intrathecal infusion, intracerebroventricular infusion, or intranasal administration since they are proven effective routes of ASO admission for crossing the blood-brain barrier $[38,42]$. On the other hand, the reticuloendothelial system limits the size of the molecules passing from the blood and lymph to the tissues [43, 44]. Unlike small therapeutic molecules, which are usually up to $500 \mathrm{Da}$ in size, the size of antisense oligonucleotides varies between $7000 \mathrm{Da}$ and $14,000 \mathrm{Da}$ [45]. This makes it difficult for them to penetrate the targeted cells and poses risks such as rapid breakdown and clearance of ASOs from the body. For this reason, carrier molecules are often attached to them. They can be viral and non-viral vectors such as lipids, liposomes, cell-penetrating peptides (CPP), and others $[39,46]$. Although viral vectors as carriers are more effective, this is not applicable with the ASO technology and especially with antisense imaging, as 
it is crucial for the ASOs to bind to the radionuclide in a convenient and rapid way for it to be absorbed by the patient due to the short half-life of the radionuclides. Lipids and liposomes are suitable carriers as they only need to be mixed with radiolabeled ASOs in order to form bonds between them. In addition, these carriers form stable bonds with negatively charged ASOs (first and second generation) and further protect them from nuclease degradation. Moreover, additional modifications in the ASO carrier, such as PEG in the lipid layer of liposomal transporters, contribute to the reduction of ASO accumulation in the reticuloendothelial organs.

Another risk in the use of radiolabeled ASOs is the fact that some radionuclides used for imaging in nuclear medicine emit low-energy electrons to varying degrees, whereby the surrounding biomolecules are also exposed to radiation [47, 48]. As a result of the use of radiolabeled ASOs targeting the cell nucleus, it is possible to cause chromosome damage and even irreparable splitting of the doublestranded DNA molecule. Examples of such radionuclides are ${ }^{125} \mathrm{I}$ and ${ }^{77} \mathrm{Br}$, as well as ${ }^{111} \mathrm{In}$. Of course, the radiation effect also depends on the radiation dose and the sensitivity of the cells, but it must still be considered when using ASOs [49]. There are methods being explored with Auger electrons emitters with working region of 10ns of nanometers [50]. Due to ASO binding with a target within the cell, the possibility of mistaken delivery and cytotoxicity in vivo remains and requires further study. However, classical Auger electron emitting radionuclides are widely used in the nuclear medicine and their physical properties, in combination with ASOs, can be used for more invasive methods such as radiotherapy.

\section{APPLICATIONS}

Despite the achievements of the ASO technology in other areas of medicine, when it comes to the role of ASOs in nuclear medicine, the potential of the technology has yet to be developed. One of the important criteria for their application in antisense imaging is to accumulate a sufficiently strong signal for radioactivity in the target [51]. For this reason, it is recommended that the target is an overexpressed mRNA, as it will have a large number of copies in the cells. Another important requirement is to minimize the radioactivity in the surrounding tissues. This is required to have a good ratio between target and non-target radioactivity to obtain a diagnostic image of high quality. However, it is also important that the dose is low enough so that there is no satiety in the target cells. On the other hand, radionuclides with a short half-life are preferred for the production of antisense imaging, which minimizes the radiation exposure of patients [52]. However, this period should not be too short in order to allow sufficient time for the ASOs to be radiolabeled, absorbed by the patient, and to reach the required ratio between target and non-target radioactivity for imaging.

Commonly used radionuclides for labelling of oligonucleotides, including ASOs, are ${ }^{3} \mathrm{H},{ }^{11} \mathrm{C},{ }^{14} \mathrm{C}$, ${ }^{14} \mathrm{~N},{ }^{32} \mathrm{P}$, and ${ }^{35} \mathrm{~S}$. The ASOs are labeled by replacing the natural isotope forms of the atoms with their radioactive variants such as ${ }^{3} \mathrm{H}$ instead of ${ }^{1} \mathrm{H}$ or ${ }^{11} \mathrm{C}$ instead of ${ }^{12} \mathrm{C}$. Such labelling is characterized by the fact that it does not affect the chemical properties of the ASO. However, for the purpose of nuclear medicine and imaging, metallic radionuclides such as ${ }^{64} \mathrm{Cu},{ }^{67} \mathrm{Ga},{ }^{89} \mathrm{Sr},{ }^{90} \mathrm{Y},{ }^{99} \mathrm{mTc}{ }^{111} \mathrm{In},{ }^{153} \mathrm{Sm},{ }^{177} \mathrm{Lu}$, ${ }^{186} \mathrm{Re},{ }^{188} \mathrm{Re}$, and ${ }^{201} \mathrm{Tl}$ are usually used [52, 53]. Most of them require the radiolabeling to be performed by means of chelates so as not to impair the chemical properties of the antisense oligomers. Examples of such chelates used in the clinical practice are DTPA, $\mathrm{MAG}_{3}, \mathrm{HYNIC}$, and DOTA.

According to Hnatowich et al. the binding of the first generation of phosphorothioated ASOs with ${ }^{111}$ In and ${ }^{99 \mathrm{~m}} \mathrm{Tc}$ is inapplicable in vivo, as the resulting radiopharmaceuticals show a strong tendency to bind to peptides in the body [54].Despite that in 2004 Zheng et al. demonstrates clear tumor images $2 \mathrm{~h}$ after intravenous administration of 15 mer first-generation ASO, radiolabeled with ${ }^{99 \mathrm{~m}} \mathrm{Tc}$ [55]. In addition, in another study from 2003 , a ${ }^{99 \mathrm{~m}} \mathrm{Tc}$ ASO, carried by liposomes, indicates successful inhibition of the c-myc oncogene expression by emitting low energy electrons that damage the target and hence it inhibits the growth of cancer cells [56].This demonstrates that in addition to the use of antisense oligonucleotides as radiopharmaceuticals for antisense images, it is also possible to use them together for antitumor treatment. In these cases, radionuclides with high LET emissions over short distances $(\alpha$-particles, $\beta$ - particles, or Auger electrons), which are highly cytotoxic, can be used for labelling with therapeutic purposes. Ou et al. examine the effect of 15-mer first generation ASO, radiolabeled with ${ }^{131} \mathrm{I}$ and carried by VIP-polylysine for the treatment of cancer cells [57].VIP- ${ }^{131}$ I-ACO targets the start region of the c-myc mRNA and successfully inhibits 9,67-fold the growth of tumor cells in athymic mice bearing HT29 tumor xenografts. 


\section{CONCLUSIONS}

The antisense technology allows any mRNA to be used as a target, especially if it is overexpressed due to the appearance of a certain pathological condition in the body such as cancer. The ASO technology has enormous potential for the specific targeting, imaging, and possible follow-up therapy in such a harmful disease. Although there are still some obstacles to be tackled, the antisense imaging and treatment will continue to benefit from the constant development of antisense technology and in particular the improvements in the chemical modifications that will lead to the further optimization of this approach. It remains to be seen whether radionuclide-labeled antisense oligonucleotides will be successful as a tool of the nuclear medicine in the near future. However, our opinion is that due to the advantages of the ASO technology it is worth the effort, both in the development of this relatively new imaging approach and in the development of radio-ASO antitumor therapy.

The TR 24 cyclotron that will be installed at the new cyclotron center of INRNE-BAS [58, 59] is a medium energy (18-24 MeV), high current cyclotron, manufactured by Advanced Cyclotron Systems, Inc., Canada. The relatively high proton beam energy compared to that of the medical cyclotrons and the high current available with this cyclotron allow to produce commercial quantities of SPECT radionuclides as ${ }^{123} \mathrm{I},{ }^{111} \mathrm{In},{ }^{67} \mathrm{Ga},{ }^{99 \mathrm{~m}} \mathrm{Tc}$ and large quantities of the traditional PET radionuclides as ${ }^{11} \mathrm{C},{ }^{13} \mathrm{~N},{ }^{15} \mathrm{O},{ }^{18} \mathrm{~F},{ }^{124} \mathrm{I},{ }^{64} \mathrm{Cu},{ }^{68} \mathrm{Ga}$. Among these classical radionuclides with applications in nuclear medicine ${ }^{123} \mathrm{I},{ }^{111} \mathrm{In},{ }^{67} \mathrm{Ga},{ }^{99 \mathrm{~m}} \mathrm{Tc},{ }^{124} \mathrm{I}$ emit also low energy Auger electrons and could find applications for targeted radiotherapy by radiolabeled ASOs. There are also several less popular and newly emerging Auger emitters that can be produced [60] with the TR 24 cyclotron such as ${ }^{165} \mathrm{Er},{ }^{119} \mathrm{Sb},{ }^{117} \mathrm{Sb}$, ${ }^{191} \mathrm{Pt},{ }^{58 \mathrm{~m}} \mathrm{Co},{ }^{135} \mathrm{La},{ }^{77} \mathrm{Br}$ and ${ }^{167} \mathrm{Tm}$. The broad range of radionuclides and specifically Auger emitters available with the TR 24 cyclotron will open up new possibilities for research and development in radiopharmacy in Bulgaria including research with radiolabeled ASOs.

ACKNOWLEDGEMENTS. This research has been supported by the Bulgarian Ministry of Education and Science trough the National Roadmap for Research Infrastructure 2017-2023 for the National Cyclotron Centre.

\section{REFERENCES}

1. Martinovich K.M., N.C. Shaw, A. Kicic, A. Schultz, S. Fletcher, S.D. Wilton, and S.M. Stick, The potential of antisense oligonucleotide therapies for inherited childhood lung diseases. Molecular and cellular pediatrics, 5(1), 2018, p. 3-3.

2. Mansoor M. and A.J. Melendez, Advances in antisense oligonucleotide development for target identification, validation, and as novel therapeutics. Gene regulation and systems biology, 2, 2008, p.275-295.

3. Hug N., D. Longman, and J.F. Cáceres, Mechanism and regulation of the nonsense-mediated decay pathway. Nucleic acids research, 44(4), 2016, p. 1483-1495.

4. Stein C.A. and D. Castanotto, FDAApproved Oligonucleotide Therapies in 2017. Mol Ther, 25(5), 2017, p. 1069-1075.

5. Post N., R. Yu, S. Greenlee, H. Gaus, E. Hurh, J. Matson, and Y. Wang, Metabolism and Disposition of Volanesorsen, a 2'-O-(2 methoxyethyl) Antisense Oligonucleotide, Across Species. Drug Metab Dispos, 47(10), 2019, p. 1164-1173.

6. Kim J., C. Hu, C. Moufawad El Achkar, L.E. Black, J. Douville, A. Larson, M.K. Pendergast, S.F. Goldkind, E.A. Lee, A. Kuniholm, A. Soucy, J. Vaze, N.R. Belur, K. Fredriksen, I. Stojkovska, A. Tsytsykova, M. Armant, R.L. DiDonato, J. Choi, L. Cornelissen, L.M. Pereira, E.F. Augustine, C.A. Genetti, K. Dies, B. Barton, L. Williams, B.D. Goodlett, B.L. Riley, A. Pasternak, E.R. Berry, K.A. Pflock, S. Chu, C. Reed, K. Tyndall, P.B. Agrawal, A.H. Beggs, P.E. Grant, D.K. Urion, R.O. Snyder, S.E. Waisbren, A. Poduri, P.J. Park, A. Patterson, A. Biffi, J.R. Mazzulli, O. Bodamer, C.B. Berde, and T.W. Yu, Patient-Customized Oligonucleotide Therapy for a Rare Genetic Disease. N Engl J Med, 381(17), 2019, p. 1644-1652.

7. Warfield K.L., D.L. Swenson, G.G. Olinger, D.K. Nichols, W.D. Pratt, R. Blouch, D.A. Stein, M.J. Aman, P.L. Iversen, and S. Bavari, Gene-specific countermeasures against Ebola virus based on antisense phosphorodiamidate morpholino oligomers. PLoS Pathog, 2(1), 2006, p. el.

8. Ge Q., M. Pastey, D. Kobasa, P. Puthavathana, C. Lupfer, R.K. Bestwick, P.L. Iversen, J. Chen, and D.A. Stein, Inhibition of Multiple Subtypes of Influenza A Virus in Cell Cultures with Morpholino Oligomers. Antimicrobial Agents and Chemotherapy, 50(11), 2006, p. 3724-3733. 
9. Spurgers K.B., C.M. Sharkey, K.L. Warfield, and S. Bavari, Oligonucleotide antiviral therapeutics: antisense and RNA interference for highly pathogenic RNA viruses. Antiviral Res, 78(1), 2008, p. 26-36.

10. Hashimoto M., T. Nara, H. Hirawake, J. Morales, M. Enomoto, and K. Mikoshiba, Antisense Oligonucleotides Targeting Parasite Inositol 1,4,5Trisphosphate Receptor Inhibits Mammalian Host Cell Invasion by Trypanosoma cruzi. Scientific reports, 4, 2014, p. 4231.

11. Marafini I. and G. Monteleone, Inflammatory bowel disease: new therapies from antisense oligonucleotides. Ann Med, 50(5), 2018, p. 361-370.

12. Southwell A.L., N.H. Skotte, C.F. Bennett, and M.R. Hayden, Antisense oligonucleotide therapeutics for inherited neurodegenerative diseases. Trends Mol Med, 18(11), 2012, p. 634-43.

13. Kordasiewicz H.B., L.M. Stanek, E.V. Wancewicz, C. Mazur, M.M. McAlonis, K.A. Pytel, J.W. Artates, A. Weiss, S.H. Cheng, L.S. Shihabuddin, G. Hung, C.F. Bennett, and D.W. Cleveland, Sustained therapeutic reversal of Huntington's disease by transient repression of huntingtin synthesis. Neuron, 74(6), 2012, p. 1031-1044.

14. Sully E.K. and B.L. Geller, Antisense antimicrobial therapeutics. Curr Opin Microbiol, 33, 2016, p. 47-55.

15. Bai H., X. Xue, Z. Hou, Y. Zhou, J. Meng, and $X$. Luo, Antisense antibiotics: a brief review of novel target discovery and delivery. Curr Drug Discov Technol, 7(2), 2010, p. 76-85.

16. Biroccio A., C. Leonetti, and G. Zupi, The future of antisense therapy: combination with anticancer treatments. Oncogene, 22(42), 2003, p. 6579-88.

17. Moulder S.L., W.F. Symmans, D.J. Booser, T.L. Madden, C. Lipsanen, L. Yuan, A.M. Brewster, M. Cristofanilli, K.K. Hunt, T.A. Buchholz, J. Zwiebel, V. Valero, G.N. Hortobagyi, and F.J. Esteva, Phase I/II study of G3139 (Bcl-2 antisense oligonucleotide) in combination with doxorubicin and docetaxel in breast cancer. Clin Cancer Res, 14(23), 2008, p. 7909-16.

18. Le B.T., P. Raguraman, T.R. Kosbar, S. Fletcher, S.D. Wilton, and R.N. Veedu, Antisense Oligonucleotides Targeting Angiogenic Factors as Potential Cancer Therapeutics. Mol Ther Nucleic Acids, 14, 2019, p. 142-157.
19. M H. and R. SN, Voraussetzungen für den Einsatz von Antisense-Diagnostika in der Nuklearmedizin [Prerequisites for diagnostic use of antisense oligonucleotides in nuclear medicine]. 35(4), 1996.

20. Iyer A.K. and J. He, Radiolabeled oligonucleotides for antisense imaging. Current organic synthesis, 8(4), 2011, p. 604-614.

21. Boado R.J. and W.M. Pardridge, Imaging gene expression in the brain with peptide nucleic acid (PNA) antisense radiopharmaceuticals and drug targeting technology. Letters in Peptide Science, 10(3), 2003, p. 169-190.

22. Dewanjee M.K., N. Haider, and J. Narula, Imaging with radiolabeled antisense oligonucleotides for the detection of intracellular messenger RNA and cardiovascular disease. J Nucl Cardiol, 6(3), 1999, p. 345-56.

23. Lendvai G., S. Estrada, and M. Bergström, Radiolabelled oligonucleotides for imaging of gene expression with PET. Curr Med Chem, 16(33), 2009, p. 4445-61.

24. Seo Y., C. Mari, and B.H. Hasegawa, Technological development and advances in singlephoton emission computed tomography/computed tomography. Seminars in nuclear medicine, 38(3), 2008, p. 177-198.

25. Shimojo M., Y. Kasahara, M. Inoue, S.-i. Tsunoda, Y. Shudo, T. Kurata, and S. Obika, A gapmer antisense oligonucleotide targeting SRRM4 is a novel therapeutic medicine for lung cancer. Scientific Reports, 9(1), 2019, p. 7618.

26. A V.-P., P. KB, and P. R, Drug Discovery for Targeting Drug Resistant Bacteria, in Sustainable Agriculture Reviews 46, P. H, S. C,L. E, Editors, Springer, Cham. , 2020, p. 23.

27. Sciabola S., H. Xi, D. Cruz, Q. Cao, C. Lawrence, T. Zhang, S. Rotstein, J.D. Hughes, D.R. Caffrey, and R.V. Stanton, PFRED: A computational platform for siRNA and antisense oligonucleotides design. PloS one, 16(1), 2021, p. e0238753-e0238753.

28. Bo X., S. Lou, D. Sun, J. Yang, and S. Wang, AOBase: a database for antisense oligonucleotides selection and design. Nucleic acids research, 34(Database issue), 2006, p. D664-D667.

29. Lorenz R., S.H. Bernhart, C. Höner zu Siederdissen, H. Tafer, C. Flamm, P.F. Stadler, and I.L. Hofacker, ViennaRNA Package 2.0. Algorithms for Molecular Biology, 6(1), 2011, p. 26. 
30. Yu R.Z., J.S. Grundy, and R.S. Geary, Clinical pharmacokinetics of second generation antisense oligonucleotides. Expert Opin Drug Metab Toxicol, 9(2), 2013, p. 169-82.

31. Niemietz C., G. Chandhok, and H. Schmidt, Therapeutic Oligonucleotides Targeting Liver Disease: TTR Amyloidosis. Molecules, 20(10), 2015, p. 17944-75.

32. Järver P., T. Coursindel, S.E. Andaloussi, C. Godfrey, M.J. Wood, and M.J. Gait, Peptidemediated Cell and In Vivo Delivery of Antisense Oligonucleotides and siRNA. Molecular therapy. Nucleic acids, 1(6), 2012, p. e27-e27.

33. Xue X.Y., X.G. Mao, Y. Zhou, Z. Chen, Y. $\mathrm{Hu}$, Z. Hou, M.K. Li, J.R. Meng, and X.X. Luo, Advances in the delivery of antisense oligonucleotides for combating bacterial infectious diseases. Nanomedicine, 14(3), 2018, p. 745-758.

34. Kang L., X.J. Xu, C. Ma, R.F. Wang, P. Yan, C.L. Zhang, H.W. Sun, and D. Li, Optimized preparation of a $(99 \mathrm{~m}) \mathrm{Tc}$-radiolabeled probe for tracing microRNA. Cell Biochem Biophys, 71(2), 2015, p. 905-12.

35. Saeidnia S., A. Manayi, and M. Abdollahi, From in vitro Experiments to in vivo and Clinical Studies; Pros and Cons. Curr Drug Discov Technol, 12(4), 2015, p. 218-24.

36. Evers M.M., L.J.A. Toonen, and W.M.C. van Roon-Mom, Antisense oligonucleotides in therapy for neurodegenerative disorders. Advanced Drug Delivery Reviews, 87, 2015, p. 90-103.

37. Miller T.M., A. Pestronk, W. David, J. Rothstein, E. Simpson, S.H. Appel, P.L. Andres, K. Mahoney, P. Allred, K. Alexander, L.W. Ostrow, D. Schoenfeld, E.A. Macklin, D.A. Norris, G. Manousakis, M. Crisp, R. Smith, C.F. Bennett, K.M. Bishop, and M.E. Cudkowicz, An antisense oligonucleotide against SOD1 delivered intrathecally for patients with SOD1 familial amyotrophic lateral sclerosis: a phase 1, randomised, first-in-man study. The Lancet. Neurology, 12(5), 2013, p. 435-442.

38. Chen Y., C. Mazur, Y. Luo, L. Sun, M. Zhang, A. McCampbell, and G.S. Tomassy, Intrathecal Delivery of Antisense Oligonucleotides in the Rat Central Nervous System. J Vis Exp, (152), 2019.

39. Juliano R.L., The delivery of therapeutic oligonucleotides. Nucleic acids research, 44(14), 2016, p. 6518-6548.
40. Geary R.S., R.Z. Yu, T. Watanabe, S.P. Henry, G.E. Hardee, A. Chappell, J. Matson, H. Sasmor, L. Cummins, and A.A. Levin, Pharmacokinetics of a tumor necrosis factor-alpha phosphorothioate 2'-O-(2-methoxyethyl) modified antisense oligonucleotide: comparison across species. Drug Metab Dispos, 31(11), 2003, p. 1419-28.

41. Geary R.S., D. Norris, R. Yu, and C.F. Bennett, Pharmacokinetics, biodistribution and cell uptake of antisense oligonucleotides. Adv Drug Deliv Rev, 87, 2015, p. 46-51.

42. Hashizume R., T. Ozawa, S.M. Gryaznov, A.W. Bollen, K.R. Lamborn, W.H. Frey, 2nd, and D.F. Deen, New therapeutic approach for brain tumors: Intranasal delivery of telomerase inhibitor GRN163. Neuro-oncology, 10(2), 2008, p. 112-120.

43. Yuan S.Y. and R.R. Rigor, Regulation of Endothelial Barrier Function, San Rafael (CA): Morgan \& Claypool Life Sciences, 2010.

44. Lee S., I. Choi, and Y.K. Hong, Heterogeneity and plasticity of lymphatic endothelial cells. Semin Thromb Hemost, 36(3), 2010, p. 352-61.

45. Shen X. and D.R. Corey, Chemistry, mechanism and clinical status of antisense oligonucleotides and duplex RNAs. Nucleic acids research, 46(4), 2018, p. 1584-1600.

46. Margus H., K. Padari, and M. Pooga, Cellpenetrating peptides as versatile vehicles for oligonucleotide delivery. Mol Ther, 20(3), 2012, p. 525-33.

47. Hnatowich D.J., Antisense and nuclear medicine. J Nucl Med, 40(4), 1999, p. 693-703.

48. Knapp F.F. and A. Dash, Auger Electron-Based Radionuclide Therapy, in Radiopharmaceuticals for Therapy, Springer India: New Delhi, 2016, p. 57-67.

49. Ku A., V.J. Facca, Z. Cai, and R.M. Reilly, Auger electrons for cancer therapy - a review. EJNMMI Radiopharmacy and Chemistry, 4(1), 2019, p. 27.

50. Riley E.A., C.M. Hess, and P.J. Reid, Photoluminescence intermittency from single quantum dots to organic molecules: emerging themes. International journal of molecular sciences, 13(10), 2012, p. 12487-12518.

51. Hnatowich D.J., Antisense imaging: where are we now? Cancer Biother Radiopharm, 15(5), 2000, p. 447-57. 
52. Liu Y., G. Liu, and D.J. Hnatowich, A Brief Review of Chelators for Radiolabeling Oligomers. Materials, 3(5), 2010, p. 3204-3217.

53. Velikyan I., M. Bergström, S. Estrada, D. Laryea, M. Välilä, S. Salomäki, B. Langstrom, and A. Roivainen, Biodistribution of Ga-68-labelled phosphodiester, phosphorothioate, and 2 '-O-methyl phosphodiester oligonucleotides in normal rats. European journal of pharmaceutical sciences: official journal of the European Federation for Pharmaceutical Sciences, 26, 2005, p. 26-38.

54. Hnatowich D.J., Pharmacokinetics of 99mTc-labeled oligonucleotides. Q J Nucl Med, 40(3), 1996, p. 202-8.

55. Zheng J.G. and T.Z. Tan, Antisense imaging of colon cancer-bearing nude mice with liposomeentrapped 99m-technetium-labeled antisense oligonucleotides of c-myc mRNA. World J Gastroenterol, 10(17), 2004, p. 2563-6.

56. Zheng J., T. Tan, W. Pan, and C. Zhang, [Study on the antisense inhibition effect of liposomemediated radiolabled antisense oligonucleotides of c- myc mRNA]. Sichuan Da Xue Xue Bao Yi Xue Ban, 34(3), 2003, p. 413-6.

57. Ou X., T. Tan, L. He, Y. Li, J. Li, and A. Kuang, Antitumor effects of radioiodinated antisense oligonuclide mediated by VIP receptor. Cancer Gene Therapy, 12(3), 2005, p. 313-320.

58.Tonev D., N. Goutev, and L.S. Georgiev, Cyclotron laboratory of the Institute for Nuclear Research and Nuclear Energy. Journal of Physics: Conference Series, 724, 2016, p. 012049.

59. Tonev D., N. Goutev, G. Asova, A. Artinyan, A. Demerdjiev, L.S. Georgiev, M. Yavahchova, V. Bashev, S.G. Genchev, E. Geleva, M. Mincheva, A. Nikolov, and D.T. Dimitrov, National cyclotron centre at the Institute for Nuclear Research and Nuclear Energy. Journal of Physics: Conference Series, 1023, 2018, p. 012035.

60. Sobolev A., R. Aliev, and S. Kalmykov, Radionuclides emitting particles with short pathway and modular nanotransporters for their delivery to cancer target cells. Russian Chemical Reviews, 85, 2016.

Assist. Dr. Katya B. Popova, biologist Maria Mincheva,

Assist. Prof. Dr. Mariya Yavahchova, Prof. Dr. Dimitar Tonev, Assos. Prof. Dr. Nikolay Goutev

Institute for Nuclear Research and Nuclear Energy

Bulgarian Academy of Sciences

72, Tsarigradsko Shosse 1784 Sofia, Bulgaria

e-mails: kate_b_popova@yahoo.com

maria.m.bg91@gmail.com

m.yavahchova@gmail.com

mitko@inrne.bas.bg

nikolay.v.goutev@ hotmail.com 